\title{
Curving performance simulation of steering bogie using magnetic elastomer
}

\author{
Y. Umehara ${ }^{1,2}$, T. Oguro ${ }^{2,3}$, S. Kamoshita ${ }^{1}$, K. Nagashima ${ }^{2,3}$, \\ S. Sasaki ${ }^{2,3}$, M. Kawai ${ }^{2,3}$ \& T. Mitsumata ${ }^{2,3}$ \\ ${ }^{I}$ Vehicle Structure Technology Division \\ Railway Technical Research Institute, Japan \\ ${ }^{2}$ Graduate School of Science and Technology, Niigata University, Japan \\ ${ }^{3} J S T-A L C A$, Japan
}

\begin{abstract}
In order to improve the curving performance of a railway vehicle, the authors have devised a steering system in which a magnetic elastomer is used for the elastic members such as a rubber bushing of the axle box suspension. This material is characterized by its hardness variation depending on the magnetic field. This steering system is capable of varying the longitudinal stiffness of the axle box suspension. In straight sections, the application of this system ensures running stability by increasing the longitudinal stiffness of the axle box suspension by means of applying a magnetic field. On the other hand, in curve sections, it improves curving performance by decreasing the longitudinal stiffness of the primary suspension by means of turning off the magnetic field. The authors made test pieces towards the development of the magnetic elastomer for the steering bogie. In a characteristic test, the authors confirmed that the Young's modulus of the magnetic elastomer changed in the range of about five times depending on the presence or absence of the magnetic field. In addition, the authors simulated the 32-degrees-of-freedom vehicle running model by applying the stiffness change of the magnetic elastomer to the steering bogie. The authors confirmed that this steering bogie was capable of reducing the mean outer wheel lateral force in the circular curve section approximately by $20 \%$ compared to that of the normal bogie.
\end{abstract}

Keywords: magnetic elastomer, axle box suspension, steering bogie, lateral force, longitudinal stiffness. 


\section{Introduction}

Increasing the longitudinal stiffness of the axle box suspension of the bogie for the railway vehicle is effective in ensuring its running stability at high speeds. However, if the longitudinal stiffness of the axle box suspension increases, the curving performance is reduced because the self-steering of the wheelset in the curve section is inhibited. Therefore, in order to improve the curving performance while ensuring the running stability, steering bogies of various types have conventionally been researched and developed. The steering bogie is a bogie for reducing the lateral force by improving the direction of the wheelset in the curve section. A conventional type of bogie angle linked steering bogie which has been used on the commercial line in Japan adopts a method of steering the wheelsets depending on the relative yawing angle between the car body and the bogie. This kind of bogie has no risk of the reverse steering, and is able to decrease the wheel lateral force very much in the circular curve section [1]. However, the steering bogie has problems such as complicated bogie maintenance work because of the complicated bogie structure equipped with steering devices such as a link. Therefore, the saving maintenance of the steering bogie by removing the heavy parts and the wear parts is required.

In this paper, the authors proposed the construction of the steering bogie by the axle box suspension using the magnetic elastomer in order to save maintenance work. Firstly, the authors show the characteristics test results of the magnetic elastomer developed for railway vehicles. Secondly, the authors report on the curving performance simulation analysis of a railway vehicle equipped with the steering bogie, with the result that the authors confirmed the effectiveness of the axle box suspension by means of the magnetic elastomer.

\section{Development of the magnetic elastomer for railway vehicles}

\subsection{Magnetic elastomer}

The magnetic elastomer is composed of magnetic particles and the elastomer such as the synthetic rubber. This material is characterized by its hardness variation depending on the magnetic field $[2,3]$. The schematic illustrations of the magnetic elastomer are shown in Figure 1. Further, applied research that exploits the phenomenon of hardness changes by a magnetic field is underway in the field of the vibration control devices of automotives [4], the haptic interface [5], the prosthetic foot [6] and the soft actuators [7]. In this study, in order to apply the magnetic elastomer to the bogie for a railway vehicle, the authors will require the synthesis of the magnetic elastomer that can demonstrate the performance of the hardness variation under the high-load condition. 


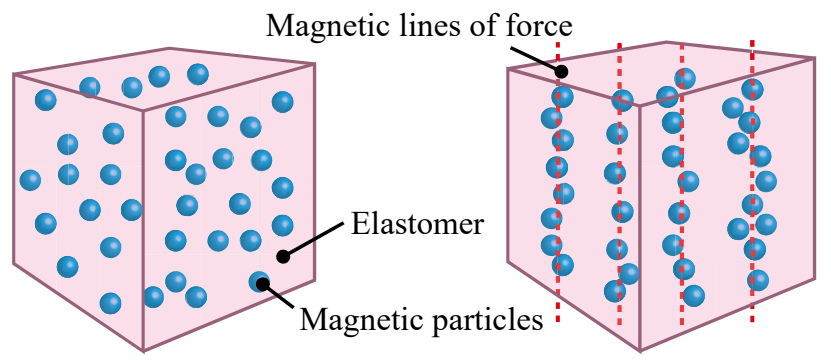

(a) Before magnetization

(b) After magnetization

Figure 1: Schematic illustrations of the magnetic elastomer.

\subsection{Proposed steering bogie using a magnetic elastomer}

In this paper, the authors proposed to apply the magnetic elastomer to elastic parts such as rubber bushings used in the axle box suspension (as shown in Figure 2). By the system configurations as shown in Figure 3, this steering system is capable of varying the longitudinal stiffness of the axle box suspension. In straight sections or high speed sections, the application of this system ensures the running stability by increasing the longitudinal stiffness of the axle box

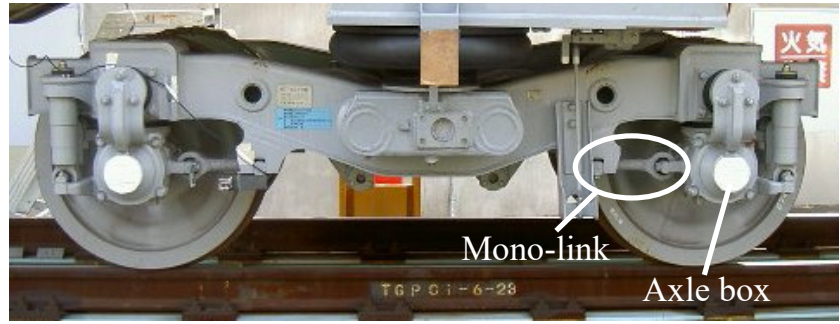

(a) Axle box suspension

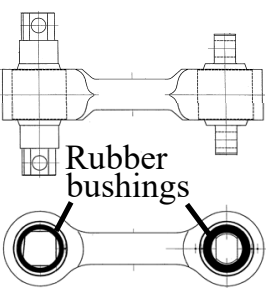

(b) Mono-link

Figure 2: Example of the axle box suspension (mono-link type).

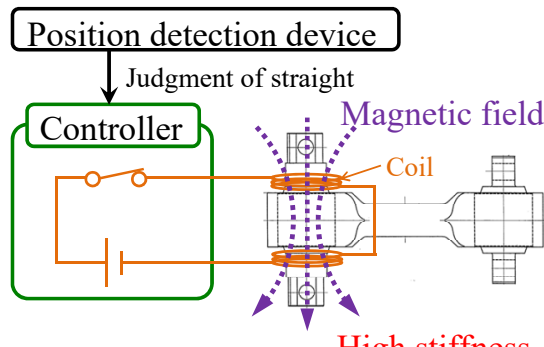

$\underline{\text { High stiffness }}$

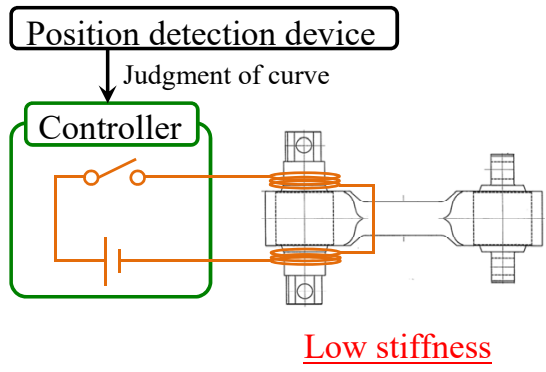

(b) Curve section

Figure 3: Schematic illustrations of the variable stiffness mono-link. 
suspension by means of applying a magnetic field. On the other hand, in curve sections or low speed sections, it improves curving performance by decreasing the longitudinal stiffness of the axle box suspension by means of turning off the magnetic field. If the railway vehicle is not equipped with a position detection device as is shown in Figure 3, it is possible to detect the curve section by using the yaw rate sensor. Further, the steering system can also determine the necessity of the magnetic field to be applied to each line by obtaining the operation codes or the stopping station information from the operation control device. Moreover, it is possible to make the magnetic elastomer further acquire the reverse characteristics by blending another elastomer and magnetic particles.

\subsection{Young's modulus changes depending on the magnetic field}

In order to develop the magnetic elastomer for rail vehicles, the authors made a test piece $(\varphi 35 \mathrm{~mm}, \mathrm{t} 10 \mathrm{~mm})$ as is shown in Figure 4, and the authors examined the change in the Young's modulus of the magnetic elastomer depending on the presence or absence of a magnetic field. Magnetic elastomer is a blend of the main material of the polyurethane and the magnetic particles of a pure iron type. The authors measured the stress by providing the strain at a compression rate of $10 \mathrm{~mm} / \mathrm{min}$ by using a material testing machine (EZ-S 500N: SHIMADZU). In addition, the magnetic field was applied by placing a permanent magnet with a surface magnetic flux density of $310 \mathrm{mT}$. Figure 5 shows the relationships between the strain and stress for the magnetic elastomer. Young's modulus was approximately $0.4 \mathrm{MPa}$ when the magnetic field is not applied, and was approximately $2 \mathrm{MPa}$ when the magnetic field is applied. Consequently, the authors confirmed the change of approximately five times in the Young's modulus by the presence or absence of a magnetic field.

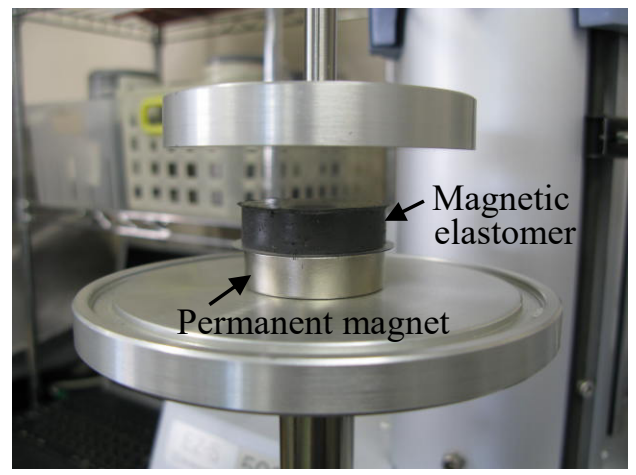

Figure 4: Young's modulus measurement of a magnetic elastomer. 


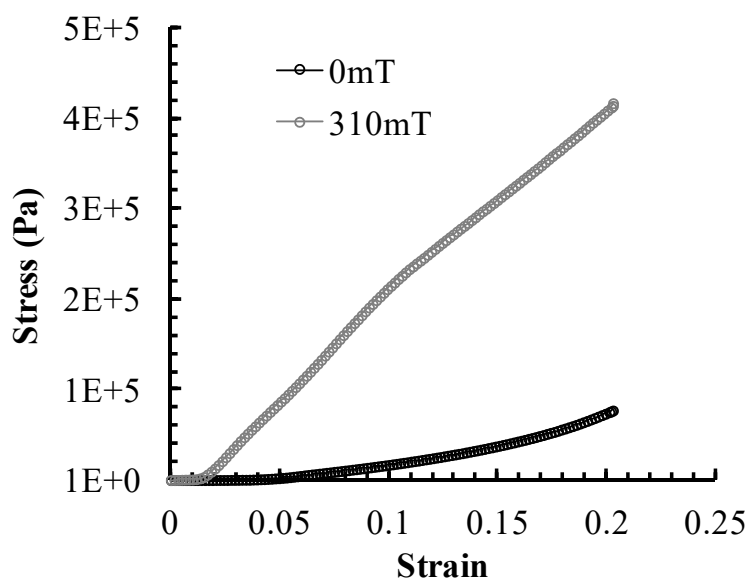

Figure 5: Strain-stress curves for the magnetic elastomer at $0 \mathrm{mT}$ and $310 \mathrm{mT}$.

\section{Evaluation by the dynamics analysis of a railway vehicle}

\subsection{Dynamics analysis model of a railway vehicle}

The authors created a railway vehicle model in which the magnetic elastomer whose rate of hardness change by the presence or absence of the magnetic field is approximately five times is used for all the longitudinal stiffening elements of the axle box suspension. Figure 6 shows the railway vehicle model used in the dynamics analysis of this paper. This analysis model had 32 degrees of freedom (DOF), including 12 DOF on the lateral, longitudinal, rolling and yawing motion of a car body and two bogie frames, 12 DOF on the lateral, longitudinal and yawing motion of four wheelsets, and 8 DOF on the lateral motion of rails under eight wheels. The authors performed the numerical analysis by using the RungeKutta-Gill method in order to solve the governing equation of this model. The authors conducted a two-dimensional geometry contact calculation of the wheel and the rail, and created a database, including the contact angle, the contact radius, the contact spacing for the lateral displacement and the rolling angle of the wheelset beforehand. Then, the analysis program was a method of referencing the database sequentially in response to the movement of the wheelset.

\subsection{Conditions of the dynamics analysis}

The authors conducted comparative evaluations by the presence or absence of a magnetic field for the lateral force generated in the curve section. Table 1 shows the main specifications of the railway vehicle, and Table 2 shows the main specifications of the curve. These values are assumed the commuter train in 
Japan. In this paper, the maximum magnetic flux density was $310 \mathrm{mT}$, and the change rate of the longitudinal stiffness of the axle box suspension depending on the presence of the magnetic field was obtained by linear approximation results in Figure 5. Conditions for applying the magnetic field were as follows: (a) not apply the magnetic field $(0 \mathrm{mT})$, (b) apply the magnetic field $(310 \mathrm{mT})$, and (c) not apply the magnetic field in the circular curve. In addition, the condition of (c) is to confirm the possibility of the steering control in the curve section.

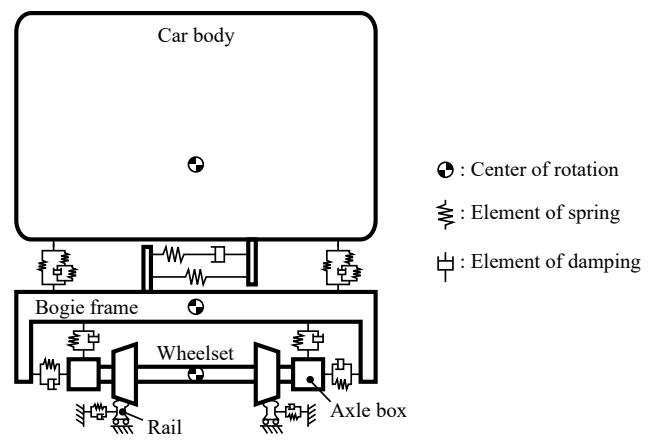

(a) Front view

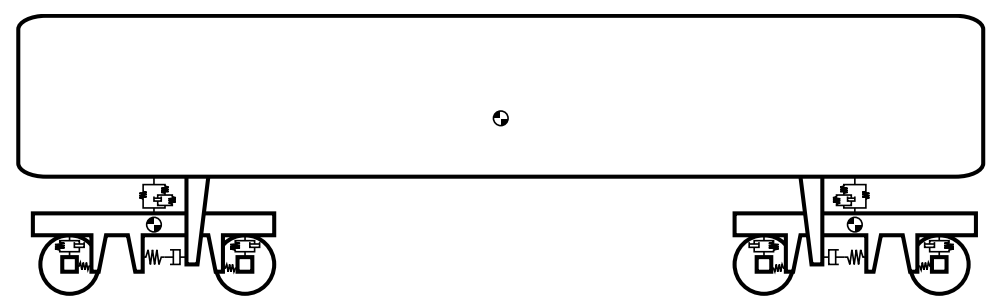

(b) Side view

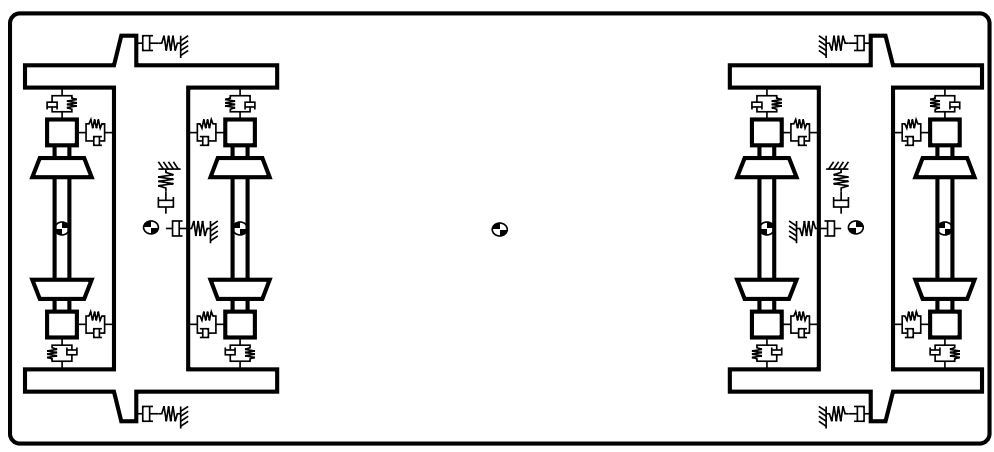

(c) Top view

Figure 6: Dynamics analysis model of a railway vehicle. 
Table 1: $\quad$ Main specifications of the railway vehicle.

\begin{tabular}{|c|c|}
\hline Distance between two bogies & $13.8 \mathrm{~m}$ \\
\hline Wheel base & $2.1 \mathrm{~m}$ \\
\hline Mass of a car body & $18820 \mathrm{~kg}$ \\
\hline Mass of a bogie frame & $2070 \mathrm{~kg}$ \\
\hline Mass of a wheelset & $1520 \mathrm{~kg}$ \\
\hline Wheel diameter & $860 \mathrm{~mm}$ \\
\hline Running velocity & $70 \mathrm{~km} / \mathrm{h}$ \\
\hline Wheel profile & modified arc \\
\hline
\end{tabular}

Table 2: Main specifications of the curve.

\begin{tabular}{|c|c|}
\hline Gauge & $1067 \mathrm{~mm}$ \\
\hline Radius of curve & $400 \mathrm{~m}$ \\
\hline Length of circular curve & $250 \mathrm{~m}$ \\
\hline Length of transition curve & $85 \mathrm{~m}$ \\
\hline Cant & $105 \mathrm{~mm}$ \\
\hline Rail profile & JIS60kg \\
\hline
\end{tabular}

\subsection{Results of the dynamics analysis}

Figure 7 shows the inner and outer wheel lateral force of the leading wheelset in the curve section. The direction in which the wheel pushes the rail to the outside is represented by a positive value of the wheel lateral force. The mean outer wheel lateral force in the circular curve was approximately $11 \mathrm{kN}$ when the magnetic field is applied $(310 \mathrm{mT})$, and was approximately $9 \mathrm{kN}$ when the magnetic field is not applied $(0 \mathrm{mT})$. Therefore, if the normal bogie was always in a high stiffness state of the axle box suspension, the authors confirmed that this proposed steering bogie was capable of reducing the mean outer wheel lateral force in the circular curve section approximately by $20 \%$ compared to that of the normal bogie.

Figure 8 shows the inner and outer wheel lateral force of the leading wheelset and the magnetic field intensity in conditions of "[c]not apply the magnetic field in the circular curve". When the steering control switched the magnetic field by the $\mathrm{ON}-\mathrm{OFF}$ control, the large fluctuations of the lateral force occurred at the time of the switching. As countermeasures for this problem, the authors found that it was possible to suppress the variation of the lateral force if the steering control was the proportional control of the magnetic field intensity with the electromagnet. Incidentally, that the stiffness of the magnetic elastomer varies depending on the magnetic field intensity is known [8]. From the above results, the authors confirmed that the steering control for changing the magnetic field can reduce the lateral force in the curve section, and the authors were able to indicate the possibility of the steering bogie by means of the magnetic elastomer. 

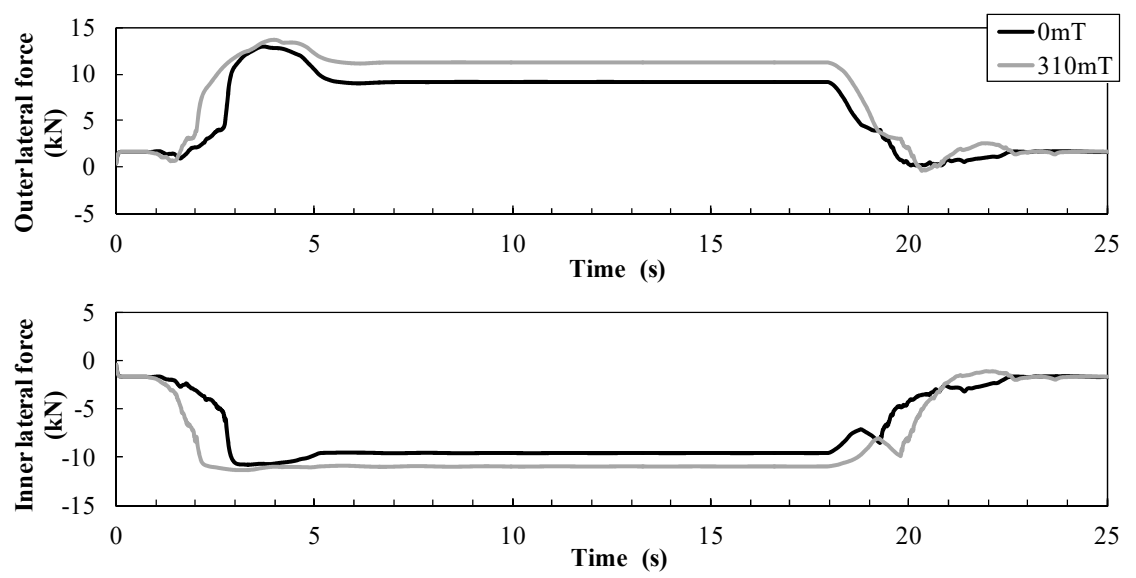

Figure 7: Simulation results of the curving performance depending on the difference in the magnetic field intensity.
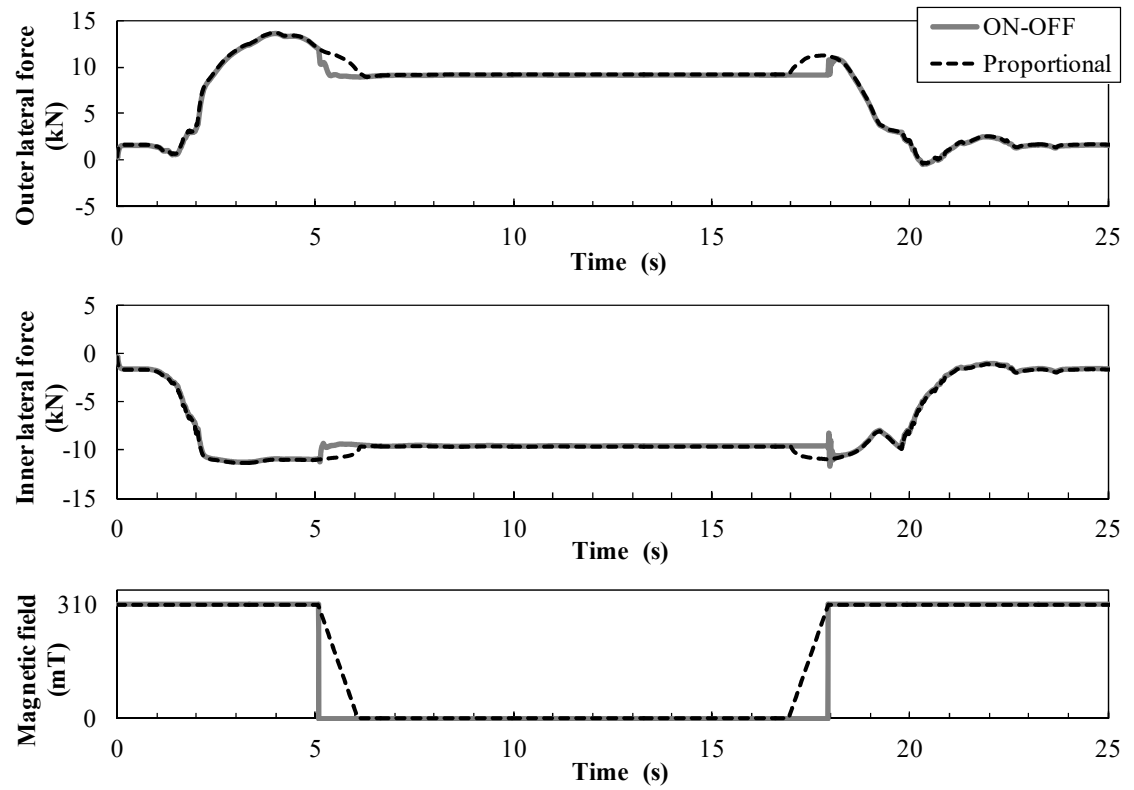

Figure 8: Simulation results of the curving performance depending on the difference in the steering control. 


\section{Conclusions}

In this paper, the authors proposed the construction of the steering bogie by the axle box suspension by means of the magnetic elastomer. Firstly, the authors made a test piece of the magnetic elastomer, and secondly, the authors examined the change in the Young's modulus of the magnetic elastomer depending on the presence or absence of a magnetic field. Consequently, the authors confirmed the change of approximately five times in the Young's modulus by the presence or absence of a magnetic field. Moreover, the authors built a dynamics analysis model of a railway vehicle, and the authors confirmed that this proposed steering bogie was capable of reducing the mean outer wheel lateral force in the circular curve approximately by $20 \%$ compared to that of the normal bogie. Henceforward, the authors will produce the cylindrical bushings using the magnetic elastomer for the purpose of evaluating the ones of railway vehicle component size.

\section{References}

[1] Sato, E. et al., Lateral Force between Wheel and Rail during Curve Negotiation of Limited Express Diesel Car with Forced Steering Bogies, Vehicle System Dynamics Supplement 37, pp. 678-689, 2002.

[2] Mitsumata, T. et al., Magnetic polyurethane elastomers with wide range modulation of elasticity, Polym. Chem., 2, pp. 1063-1067, 2011.

[3] Mitsumata, T. et al., Magnetism and viscoelasticity of magnetic elastomers with wide range modulation of dynamic modules, Soft Matter, No.9, pp. 904-912, 2013.

[4] Ginder, J.M. et al., Magnetorheological Elastomers: Properties and Applications, in Smart Structures and Materials: Smart Materials Technologies, pp. 131-138, 1999.

[5] Kikuchi, T. et al., Haptic Interface with Magnetic Field Sensitive Elastomer, Journal of Mechanics Engineering and Automation, No.3, pp. 146-151, 2013.

[6] Jonsdottir, F. et al., Preparation and Characterization of a Prototype Magnetorheological Elastomer for Application in Prosthetic Devices, ECCOMAS SMART 2015, 2015.

[7] Tsumori, F. et al., Development of actuation system for artificial cilia with magnetic elastomer, Japanese Journal of Applied Physics, 54, 06FP12, 2015.

[8] Böse, H. \& Röder, R., Magnetorheological Elastomers with High Variability of Their Mechanical Properties, J. Phys. Conf. Ser.149, 2009. 